\title{
LÉGISLATION
}

\section{Les concessions d'énergie électrique et les accidents qui leur sont reprochés. - Concordance entre plusieurs arrêts récents}

\author{
par Paul Bougnult, Avocal à la Cour d'Appel de Lyon.
}

\begin{abstract}
On croit généralement que les questions de compétence sont seulement destinées de orruper l'spril des jurisconsultes. Il n'est pas sans intérêt de faire remarquer que, dans bien des cas, la nalure d'une instance est très nettement caractérisée, par le seul fait qu'elle doit être portéc devent le Tribunal cibil; a cette juridiction sont tout spécialement réservées les affaires dans lesquelles on remarque, à la charge du distributeur, une faute ayant l'allure d'un délit (prévu par le Code pénal; par exemple homicide ou blessures par imprudence). Au contraire, la juridiction adminislrative est rissorve auz instances dans lesquelles on reproche d̀ un ouvrage public d'être mal entretenu ou mal placé, sans que la responsabilité d'une personne soit engagée au point de vue répressif.
\end{abstract}

Division de cette étude. - Pour plus de clarté, nous diviserons notre étude en deux parties, en consacrant la première aux affaires dans lesquelles l'incompétence du Tribunal judiciaire avait été formellement soulevée par le concessionnaire assigné, à raison de sa responsabilité pécuniaire, par la victime d'un accident; dans la seconde, nous examinerons les cas ou le concessionnaire a paru se désintéresser de la question de compétence.

\section{Prenière Partie:}

DÉCISIONS DANS LESQUELles LA QUESTION DE COMPÉTENCE ÉTAIT POSÉE.

Examen de deux décisions en apparence opposées. -. Leur parfaite concordance. - Deux arrêts de la Cour de Cassation qui seront reproduits ci-dessous et sont relativement rapprochés l'un de l'autre, ont été rendus l'uu par la Chambre des Requêtes, le 2 mai 1927 (affaire Casanova), l'autre par la Chambre civile, le 26 décembre 1927 (affaire Veuve Buguet-Billard) : ils ont paru être tout particulièrement en complète opposition. Le premier, en effet, en rejetant le pourvoi de la Compagnie Centrale d'Eclairage contre l'arrêt de la Cour d'Alger du 30 décembre 1925, a proclamé la compétence judiciaire et rejeté les conclusions du distributeur qui demandait à n'être jugé que par la juridiction administrative, dans la question de responsabilité pécuniaire à l'occasion d'un accident (1).

(1) On trouvera l'arrêt de la Cour de Cassation dans le Recueil de Dalloz Hebdomadaire, année 1927, page 301 (Compagnie d'Eclairage et de Chauffage par le Gaz Lebonnet Cie contre époux Casanova) :

"La Cour; sur le moyen unique pris du principe de la séparation des pouvoirs de l'article 4 , paragraphes 1 et 4 de la loi du 28 Pluviôse an VIII, et de l'article 7 de la loi du 20 avril 1810 ;

"Attendu que les époux Casanova, ayant été atteints de brûlure et de blessures par des décharges électriques provenant des fils d'éclairage installés dans leur maison par la C'ompagnie Lebon, pour la fourniture de la lumière électrique, ont introduit devant le Tribunal Civil d'Alger, une action en réparation du préjudice qui leur a été ainsi causé; que la Compagnie Lebon a prétendu et prétend encore, à l'appui de son pourvoi, que la Juridiction civile est incompétente pour connaître d'une pareille action, parce que
Le second, au contraire, a cassé un arrèt de la Cour d'Appel de: Paris du 4 janvier 1926 et a proclamé, à la demande de la Société de distribution de l'Ouest, l'incompétence du Tribunal judiciaire pour statuer sur la même responsabilité (1).

le dommage est la conséquence de la chute d'un poteau supportant une canalisation électrique et est imputable a lexécution d'un travail public;

"Mais attendu qu'il résulte des constatations de l'arrêt attaqué que la ligne sur laquelle s'est produit l'accident a été mise en service sans qu'ait été délivrée l'autorisation préfectorale de circulation de courant prévue par l'article 15 de la loi du 15 juin 1906 et qu'ainsi l'accident résulte d'une infraction aux prescriptions édictées dans l'intérêt de la sécurité des personnes et réprimée pénalement par l'article 25 de ladite loi ;

"Attendu que cette infraction ouvrait, en vertu de l'article 3 du Code d'Instruction criminelle, une action civile en dommagesintérêts devant les Tribunaux Civils; qu’en décidant dès lors que la juridiction civile était compétente pour statuer sur le litige qui lui était déféré, la Cour d'Alger n'a pas violé les textes invoqués par le pourvoi. Par ces motifs : rejette.....".

(1) Cet arrèt du 26 décembre 1927 (affaire Buguet-Billard contre Société de Distribution d'Electricité de l'Ouest) a cassé un arrêt de la Cour d'Appel de Paris du 4 janvier 1926; la Cour Suprême s'exprime ainsi (Recueil Hebdomadaire de Dalloz, année 1928, $N^{\circ} 5$, page 65 ) : La Cour. - Sur le premier moven; vu l'article 4 de la loi du 28 Pluviôse an VIII.

"Attendu que les travaux exécutés sur le territoire d'une commune par la Compagnie concessionnaire de la listribution d'énergie électrique, pour l'installation de ses lignes aćriennes, ont le caractère de travaux publics ;

"Attendu que la juridiction administrative est compétente pour statuer sur les clommages causés par l'exćcution des travaux publics ;

"Attendu que, d'après l'exploit introductif d'instance, l'accident dont a été victime le sieur Buguet et dont il était demandé réparation par sa veuve, serait le résultat d'une commotion éprouvée pendlant qu'il travaillait à la charpente d'une maison en construction, au contact de fils électriques a haute tension établis à une si faible distance du toit de l'immeuble que leur installation rendait nécessairement dangereux tout travail exécuté sur le bord de ce toit; que l'action en responsabilité engagée contre la Compagnie défenderesse, était done directement et uniquement fondée sur une prétendue défectuosité dans l'exécution d'un travail public;

"Attendu, dès lors, que c'était à l'Antorité administrative 
L'opposition n'est qu'apparente; car, en réalité, les deux problèmes à résoudie étaient loin d'être semblables.

Examinons le premier arrêl (2 mai 1927) : les époux Casanova se plaignaient d'avoir été gravement brûlés alors qu'ils étaient dans leur chambre, par le contact de leur lampe électrique dont les fils avaient momentanément rencontré des câbles à haute tension, par suite de l'affaissement d'un poteau renversé sur la route par le chauffeur de la Compagnie anonyme des Transports automobiles.

Sans doute, une part de la responsabilité devait incomber à cette dernière Société qui a d'ailleurs été maintenue en cause.

Mais, l'autre part n'incombait-elle pas à la Compagnie concessionnaire ?

Celle-ci, pour te moment du moins, ne disculait pas ce point : (1le proposait seulement it la Cour d'Alger un moyen de défense, plus exactement une fin de non recevoir que l'on peut ainsi résumer : "Si je suis responsable, ce n'est pas au Tribunal judiciaire " à le proclamer ; je suis concessionnaire d'une distribution d'éner" gie, par conséquent d'un travail public : je ne puis répondre de " mes actes que devant la juridiction administrative; car si la "Loi de Pluviose, an VIII, n'a confié aux Conseils de Préfecture " que le soin de juger la question des dommages provenant des "travaux publies, la jurisprudence depuis longtemps a étendu sa " compétence aux accidents. "(1).

Comme le remarque la Cour Supreme, les Conseillers d'Alger avaient répondu que la responsabilité de la Société concessionnaire ne dépendait pas seulement d'une bonne ou nauvaise exécution des ouvrages de la distribution, mais d'une infraction aux prescriptions qui sont imposées au distributeur pour la sécurité des personnes, et que si le distributeur s'en affranchít, il

qu'il appartenait d'en comaitre: d'ou il suit qu'en déclarant les 'I'ribunaux judiciaires compétents pour y statuer l'arrêt attaqué a meconnu le principe de la séparation des pouvoirs et violé le texte ci-dessus visé. Par ces motifs et sans qu'il soit nécessaire de statuer sur le deuxicme moyen, casse et renvoie devant la Cour d'Appel d'Orléans. "

(1) La loi de Pluxiose an VIII n'envisage expressément comme rentrant dans Ja compétence des Conseils de Préfecture que les "dommages "; mais la jurisprudence assimile à ce préjudice causé à une proprićté l'accident causé à une personne, défaut d'éclairage d'me tranchée provoquant la chute l'un passant, rupture et chute d'un fil électrique; pour le Conseil d'Etat cette assimilation ne fait pas de doute et des que la juridietion administrative est saisie, il admet la compétence administrative à la condition, bien entendu, que l'accident ne dérive pas d'un fait d'exploitation, mais soit réllement dì à l'ouvrage public lui-même; ainsi resterait dans la competence de la juridiction judiciaire un accident survenu à une femme tombant d'un tramway, parce que la plateforme n'était pas munie d'une chaine de protection (Tribunal des Conflits, 31 mai 1913; aff. Riby, Recueil du Conseil d'Etat, page $606)$; et il y aura, au contraire, compétence atministrative si raccident est dù à une fansse mancuvre exécutee pendant le cours d'un travail public : ouverture intempestive et maladroite d'une vanne par un agent d'un entrepreneur des réparations à un canal (Conseil d'Etat, 30 mars 1906; Société Métallurgicue d'Onnaing contre Carré D. P. 1907.3.19); insuffisance de solidité d'une clôture (Conseil d'letat. 7 août 1917; Recueil page 631; affaire Magnat contre Socicté Privat frères, entrepreneurs de travaux publics). La (Cour rle Cassation (voir .J. Appleton, page 425) a paru resister, pendant un certain temps, à cette opinion : elle parait aujourd'hui completement s'y rallier, sauf, comme il a été dit, le cas ou se révèle la faute constitutive d'un délit qui aurait été susseptible d'être déféré au Tribunal Correctionnel; Je document qui nous renseigne le plus fidelement sur la tendance aujourd'hui presque complète de la Cour de Cassation a admettre la eompétence administrative au sujet des aceidents, est l'arrêt qui sera analysé plus loin du 26 décembre 1927 (Buguet-Billard contre Socsété de l'Ouest, D. H. 1928, page 65). tombe sous le coup de l'article 25 de la loi du 15 juin 1906 ainsi conçu : "Toute infraction aux dispositions édictées dans l'intérêt " de la sécurité des personnes, soit par les règlements d'adminis"Lration publique, soit par les arrètés visés à l'article 19, sera " poursuivie devant les tribunaux correctionnels et punie d'une " amende de 16 francs à 3.000 francs, sans préjudice de l'applica" tion des pénalités prévues au Code pénal en cas d'accident " résultant de l'infraction. (1), "

Evidemment, on ne saurait trop le remarquer, la Société n'était pas traduite devant les tribunaux correctionnels, mais devant eux une poursuite aurait pu être engagée, et la jurisprudence est inflexible sur ce point : les juges du 'Tribunal civil sont seuls compétents pour statuer sur la responsabilité pécuniaire dérivant d'un fait qu'is auraient pu avoir ả punir comme juges correctionnels (1).

Telle est la raison pour laquelle la Cour de Cassation s'est refusée à casser l'arrèt de la Cour d'Alger qu'elle a au contraire, approuvé dans les termes que nous avons fait connaître. La jurisprudence est d'ailleurs constante : c'est la première fois que la Cour de Cassation, a notre connaissance du moins, est amenée à citer, pour une question de responsabilité pécuniaire, l'article 25 de la loi du 15 juin 1906 qui punit d'amende celui qui commet une infraction aux règlements édictés dans l'intérêt de la sécurité des personnes. Mais, elle a bien souvent cité les textes du Code pénal relatifs à l'homicide ou aux blessures par imprudence, soit contre les auteurs du fait (arrêt du 27 novembre 1918, D. P. 1919 1.17 ; Somerville contre Société des Tramways de Nice ; et 24 jan. vier 1923, 1). P. 1923.1.33, de Champlouis contre Compagnie des Tramways du Loiret), soit contre un patron responsable du fait d'un de ses employés.

Dans le cadre de cette décision, rentrent plusieurs arrêts qui ont statué sur la responsabilité du distributeur, sans que la question de l'incompétence du Tribunal judịciaire ait été soulevée : mème si elle l'avait été, d'ailleurs, la Cour aurait sûrement rejelé le déclinatoire et consacré la compétence judiciaire, pour les mo. tifs que nous avons donnés. On peut citer l'arrèt de la Chambre

(1) Ces infractions éta ient les suivantes ; ligne mise en service avant l'antorisation de courant prévue par l'article 15 de la loi du 15 juin 1906 ; mise sous tens ion sans que les précautions suffisantes aient été prises a raison d $u$ rapprochement des conducteurs de basse et de haute tension.

(1) Ce principe a été mis en pleine évidelyce, surtopt en ce quj concerne la prescription, par différents arrêts qui sont résumés dans le Répertoire Pratique de Dalloz, Ve Responsabilité Civile No 606. "Laction civile en responsabilité ne se prescrit que par " trente ans; toutefois, lorsqu'elle est fondée sur un crime, un " délit, une contravention préyus par la loi pénale, l'action civile "en responsabilité se prescrit par le mème laps de temps que " l'action publique. Mais ceci n'est vrai que si l'action civile en " responsabilité est exclusivement fondée sur des faits péprimés " par la loi pénale. Elle est, indépendante de l'action publique " neée d'un crime, d'un délit ou d'une contravention, toutes les " fois qu'elle n'a pas exclusivement pour base ce crime, ce délit. " ou cette contravention; mais çue en dehors du fait préxu par lo " loi pénale, il est relevé une autre eirconstance de laquelle puisse "découler la responsabilité de l'article 1382 du Code Civil. *

En ce qui concerne l'Etat (voir loco citato, No 609) la prescription de l'action publique à l'égard de l'auteur d'un délit n'influe en rien sur l'action en responsabilité exercée contre ledit Etat à l'occasion de ce délit, cette dernière action étant fondée non sur l'existence d'un fait délictueux, mais sur les principes suivant lescfuelles la responsabilité de l'Etat est engagée. (Consulter sur ce point Conseil d'Etat 22 mai 1912, D. P. 1915,3.3, affaire dame Augé ; 22 novembre 1918 , D. P. 1920.3 .1 , affaires diverses; consulter la note d'Appleton sur ces arrêts, D. P. 1920.3.5, paragraphe 5 in fine). 
rivile du 10 novembre 1921 (Société d'blectricite de Dòle contre Venve lissel) dans lequel la cour constate que la Société après avoir reconvert dime substance isolanle un fil donl la proximité netait pas dangereuse, na pas maintemu ce fil en bon etal d'isolement: la responsabilité dérivait d'une infraction à une mesure imposée pour la sécurité des persomes On peul consulter aussi larrèt de la Cour d'Appel de lyon du 30 octobre 1924: la Cour a ordomné une enquete pour savoir si les fils electriques arant causé la mort de louvrier charpentier Nercier etaient à une dislance réglementaire; enfin larrèt de la Cour de Montpellier du 21 juin 1924 (Société Électrique Ecoiffier contre la fille mineure Félicie Fermandez) est à rapprocher également; la cour a admis que, pour une Société de distribution, le fait de laisser trainer pendant plus de douze heures, sur la lore, un fil rompu par l'orage, engageait sa responsabilité cu vertu de larlicle 1384 du Code civil.

Examinons maintenant le second arrèt, celui du 26 décembre 1927 : l'affaire de la Vve Buguet-l3illard terminée par cet arrèt, se présentait de la facon suivante : louvrier Buguet travaillant à la charpente d'une maison en construction fut blectrocuté au contact de fils a hate tension appartenant a la Sucieté d'Electricité du Sud-Ouest; la veuve prétendit que la proximité de tals fils était trop grande et que tout travail de charpente dans ces conditions était dangereux. Mais, elle n'alléguait pas contre le distributeur un manquement nettement défini à une prescription légale édictée dans l'intérèt des personnes, telle que la distance imposée aujourd'hui par l'article 64 de l'arrèté technique pris annuellement par le Ministre des Travaux publics (le dernier paru est actuellement celui du 30 avril 1927 (1).

La demanderesse était confiante dans la facilité avec laquelle les tribunaux admettent en droit que le détenteur d'une chose est responsable des accidents causés par cette chose (article 1384 du Code civil) et, en fait, que le concessionnaire d'une distribution est soumis à une règle semblable, puisqu'il est détenteur des câbles électriques; elle espérait que le Tribunal lui ferait l'application de ce principe, d'après lequel l'observation mème stricte des règlements n'est pas une cause d'exonération de la responsabilité du distributeur (2). La Cour suprème n'a pas refusé à la demanderesse le droit de faire toutes critiques contre l'établissement des ouvrages de la Société concessionnaire; elle a simplement, sur les conclusions expresses de la Société, remarqué (et il n'en pouvait être autrement) que de pareilles critiques étaient adressées à un travail public et que le Conseil de Préfecture était seul compétent pour en connaître.

\section{SEconde ParTe}

EXAMEN DES INSTANCES DANS LESQUULLLS LA OUESTION DE COMPÉTENCE N'ÉTAT' PAS SOULEVÉE.

Arrêt de la Chambre des Requêtes du 28 novembre 1927. Quand on expose les principes que nous venons de préciser, à des

(1) Cet arrêté fixe une zone de protection déterminée par un plan vertical, parallèle au mur de façade; par un plan parallèle au toit en pente; par un plan horizontal parallèle au toit en terrasse. Les distances de ces plans par rapport aux façades, murs et toits que l'on veut garantir est de 3 mètres pour une ligne équipée avec isolateurs rigides, et de 4 mètres s'il existe des isolateurs suspendus.

(2) Voir ci-contre nos observations sur ce point. persomes gui préférent ne: pas les écouler, on se heurle souvent a l'objection qu'elles formulent d'un air plus on moins distrait : "Cependant, disent-clles, il existe an moins tun arret dans lequel "La Cour de Cassation s'est declare competente pour juger un " cas dans lequel un concessionnaire était assigné en réparation " d’un accident : il s’agissat bien d'un travail public ol aucune " latute délieluelle n’écil allegué contre le distributeur; par "conséquent d’après les principes exposés dans volre première " partie, la Cour aurail dû proclamer l'incompétence de la juri" diction judiciaire suivant en cela la doctrine qu'elle a appli"què dans l'arrèt du 26 décembre 1927 (allaire Buguel-Billard)."

Láarèt qui est particulierement visé est celui qui a été rendu le 28 novembre 1927, dans l'allatire Veuve Léonard contre Compangie linvaise d'ilectricile el est reproduit clans le Recucil Hebdomadaire de Dallo\%, ance 1928, page 5. Ia Veuve Leonad avait assigné la Compagnie Iavraise en reparation pécuniaire de lélectroculion de son mari el la Cour de Rouen l'avait déboutée de sa demande, suivant arret du 14 janvier 1924.

Constatons, tout d'abond, que la Compagnie Ilavraise n'avait déposé aucune conclusion sur la question de competence, el, dès Je début du proces, cllo avait acceplé de plaider devant la juridiction judiciaire. On, la Cour Supreme "n'affirme resllement la compétence judieinire "que dans les cas où cetle question a élé discutee devant elle. Sans doute, dans certaines hypothèses la Cour pent soulever d'office lexception d'incompélence. Mais, il serait téméraire de considérer comme bouleversant une jurisprudence, un arrêt rejelant un pourvoi contre une decision d'un tribunal judiciaire, simplement parce qu'en dehors de toul moyen soulevé par un des inleressés, il n'aurail pas invoqué doffice le principe de l'incompétence.

Et, non seulement la Compagnie Havraise n'avait pas agité cette question, mais elle arait un interêt incontestable a obtenir d'emblée une décision délinitive qui ne pouvait que lui ètre favorable, étant donné les circonstances dans lesquelles l'affaire se présentait.

(n) ne satuail oublier, en effet, que la Veuve Léonard avail perdu son procès devant la Cour de Rouen qui, pour rejeter la demande, s'était basée sur ce que les fils meurtriers étaient placis dans la situation vouluc par les règlements administratifs. Ia demanderesse au pourvoi pouvait donc présenter à la Cour Suprème cet argument bien connu : la Compagnie est détentrice de son réseau qui est sous sa garde; donc, si le réseau est la cause d'un accident la Société est en présomption de faute, en vertu de l'article 1384 ; il importe peu qu'elle ait pris toutes les précautions édictées dans des textes administratifs : la parfaite conformité aux dits textes n'est pas une cause d'exonération de responsabilité et ne supprime pas l'application de l'article 1384 du Code civil (1).

(1) Co principe est en effet constant : il surprend souvent les industriels qui allèguent toujours, pour leur défense, qu'ils ont suivi les prescriptions réglementaires ; mais les Tribunaux qui ont à statuer entre un particulier complètement étranger à la chose que le propriétaire a sous sa garde, et le propriétaire qui profite de cette chose, ne se laisscnt point toucher par cette considération que toutes les précautions légales ont été prises. Ainsi, une machine à vapeur éclate et détériore un mobilier, sans que l'on arrive à connaître le motif de l'explosion; la Compagnie se défend en disant qu'elle avait expérimenté la résistance de sa machine, en procédant aux épreuves de tension. Ia Cour de Cassation rejette ce moyen (arrêt de la Chambre Civile du 21 janvier 1919, D. P. 1922.1.25, affaire Compaguie des chemins de fer de l'Ouest contre demoiselle Marcault); mène observation dans l'arrêt de la cour the Toulouse du 11 juin 1913 (D. P. 1914.2.174, affaire Société Tou- 
Mais, dans l'arrếl de la Cour de Rouen, on ne Lrouvail pas, comme motif du rejet de la demande de la Veuve Léonard, gue la simple affirmation que la Compagnie s'était conformée aux dispositions réglementaires. La cour ajoutait un motil de grande importance: : elle constatait que la victime avait commis ellemene une faule dans le transport de la planche, avec laquelle elle avait heurté le fil électrque el avait été électrocuté ; or, si, comme nous l'avons maintes fois répété, l'arlicle 1384 mot en présomplion de faute le détenteur de la chose qui a causé l'accident et le préjudice, cetle présomption cesse quand le propriétaire de la chose démontre que la victime a commis une imprudence ou une fante.

C'est pourquoi le texte de l'arril du 28 novembre 1927 est tres interessant a lire (1).

lousaine du Bazacle contre Société I.e Phénix), arrêt ou on lit les termes suivants : "Il ne suffit pass is la Société, pour s'exonéver, de clire qu'elle a maintenu ses câbles à la distance prescrite. "

Quelques personnes demandent alors quel intérêt le concessionnaire peut trouver à se conformer aux prescriptions réglementaires, puisque, mème en les observant, il ne s'exonère pas ; il faut. d'abord remarquer que cette conformité aux règlements lui assure l'impunité au point de vue pénal ;e plus, en soulevant l'incompétence de la Juridiction judiciaire, il peut obtenir son renvoi devant la juridiction administrative qui est généralement considérée comme moins rigoureuse en ce qui concerne le chiffe des réparations pécuniaires allouées aux victimes.

(1) On peut citer certains arrets qui ont basé la responsabilité chu distributenr aussi bien sur l'article 138 \& que sur l'article 1382 du Code Civil : de ce nombre, est l'arrèt de la Cour d'Appel de Besancon du 21 mars 1927 (affaire dame Blanchard contre Société (les Grands Moulins de Gray, D. H. 1927, page 279) : cet arrèt a cassé la décision des premiers juges et décidé qu'en l'absence d'une faute de la victime, l'article 138 - était applicable en matière de canalisation électrique ; et, d'autre part. elle a trouvé que la Sisciété distributrice avait nettement commis une faute caractérisée par ce fait : six jours après laccident circulait encore dans un fil qui aurait dù rester neutre, un courant qui était supérieur à 500 volts et qui provenait du contact du réseau secondaire et du réseau primaire.
"La Cour :

"Sur le moyen unique pris de lat violation de larlicle 7 de la loi du 20 arril 1810, défaut de motifs, contradiction de motifs, manque de base legale, et cles articles 1382 et 1381 du Code (ivil ;

" Allendu que Léonard, entrepreneur de couverture à Barenlin, ayant trouvé la mort au contact de câbles installés dans cette ville pour le transport de la force electrique par la Compagnie Havraise d'Energie Electrique, et cette Société ayant élé assignée par la Vcuve Léonard, en dommages-intérêts, comme responsable de cet accideni, l'arrèt atlaqué a débouté cette dernière des fins de sa demande;

"Attendu qu'il est reproché au dit arrêt d'avoir ainsi statué, en se fondant uniquement sur ce que les fils électriques étaient placés selon les prescriptions réglementaires, alors que le respect des dites prescriptions laisse entière la responsabilité du concessionnaire vis-à-vis des tiers, et qu'il résulte des circonstances de l'accident qu'en posant des fils à haute tension assez rapprochés des maisons pour qu'une personne effectuant à celles-ci des travaux normaux, se plaçât sous la dépendance du risque, la Compagnie avait commis une faute ayant contribué à occasionner l'accident.

"Mais attendu qu'il résulte tant de l'arrèt attaqué que du juge ment du Tribunal Civil de Rouen dont il a adopté les motifs, que, contrairement à ce qui est soutenu par le pourvoi, l'accident est uniquement dâ à un mouvement maladroit de I éonard, alors que travaillant sur un échafaudage dressé à proximité des fils, il portait une planche humide qui les a heurtés;

"Attendu qu'en décidant, en cet état, des faits souverainement constatés que la Compagnie Havraise d'Energie Electrique était dégagée de toute responsabilité, larrèt attaqué qui esl motivé et dont les motifs ne contiennent ancune contradiction a légalement justifié sa décision, sans violer aucun des texles visés au moyen ;

"Par ces motifs, rejette le pourvoi. " 